\title{
High dielectric constant in charge-ordered $\mathrm{Ca}_{1.75} \mathrm{Pr}_{0.25} \mathrm{MnO}_{4}$
}

\author{
J Mira ${ }^{1}$, A Castro-Couceiro' ${ }^{2}$, M Sánchez-Andújar ${ }^{2}$, \\ B Rivas-Murias ${ }^{1}, \mathrm{~J} \mathrm{Rivas}^{1}{ }^{2}$ and M A Señarís-Rodríguez ${ }^{2}$ \\ ${ }^{1}$ Departamento de Física Aplicada, Universidade de Santiago de Compostela, \\ 15782 Santiago de Compostela, Spain \\ ${ }^{2}$ Departamento de Química Fundamental, Universidade da Coruña, 15071 A Coruña, Spain
}

Received 5 October 2005, in final form 17 January 2006

Published 3 March 2006

Online at stacks.iop.org/JPhysD/39/1192

\begin{abstract}
We report the dielectric response of $\mathrm{Ca}_{2-x} \mathrm{Pr}_{x} \mathrm{MnO}_{4}(x=0$ and 0.25).

Specifically, their intrinsic dielectric constants $\left(\varepsilon_{\mathrm{r}}^{\prime}\right)$ are calculated by impedance spectroscopy from the values of the obtained bulk capacitances. For $\mathrm{Ca}_{2} \mathrm{MnO}_{4}$, that does not show charge-order, the measured intrinsic dielectric constant is 14. The Pr doping induces charge-order in the system, and also a noteworthy increase in $\varepsilon_{\mathrm{r}}^{\prime}$, that reaches a value of $\sim 220$ for $x=0.25$, unusual in these kind of oxides. This result provides a new link between both phenomena.
\end{abstract}

\section{Introduction}

Manganese perovskites have become a marvellous natural laboratory where solid-state scientists can test multiple phenomena [1]. Among them, Jardón et al [2] and Rivadulla et al [3] reported a remarkably high capacitive response in the prototypical charge-ordered $(\mathrm{CO})$ compound $\mathrm{Pr}_{2 / 3} \mathrm{Ca}_{1 / 3} \mathrm{MnO}_{3}$, confirmed later by Saraf et al [4] on epitaxial thin films and by Mercone et al [5] in a single crystal of the same compound.

Later on, the community working on dielectric properties was surprised by the announcement of the finding of a colossal dielectric constant (CDC) in a non-ferroelectric material [6]. The debate soon reached the conclusion that the reported $\mathrm{CDC}$ was mainly due to extrinsic effects [7-9]. Nevertheless, the fact that in $\operatorname{Pr}_{2 / 3} \mathrm{Ca}_{1 / 3} \mathrm{MnO}_{3}$ the high capacitive response disappears just beyond the charge-ordering transition temperature, $T_{\mathrm{CO}},[2-4]$ points to a clear influence of the $\mathrm{CO}$ state, irrespective of the extrinsic factors that might play a role. It seems then plausible to think of an influence of the electronic state on the dielectric response of a solid. Given that perovskite-type manganites exhibit 3D character, to modify and understand their properties it is worth reducing a dimension. In order to do this, and in order to test the CO hypothesis, we have focused on the two-dimensional version of (Pr,Ca) $\mathrm{MnO}_{3}, \mathrm{Ca}_{2-x} \mathrm{Pr}_{x} \mathrm{MnO}_{4}$, a system which exhibits a longrange charge-orbital ordering state [10] over the composition range $0.25 \leqslant x \leqslant 0.5$, and at relatively high temperatures $\left(270 \leqslant T_{\mathrm{CO}}(\mathrm{K}) \leqslant 330\right)$. In addition, the high resistivity of these compounds reduces the role of extrinsic effects on the determination of the dielectric constant, extrinsic effects that are quite important in the measurement of the dielectric constant of $\mathrm{Pr}_{2 / 3} \mathrm{Ca}_{1 / 3} \mathrm{MnO}_{3}$ [5].

\section{Experimental}

$\mathrm{Ca}_{2-x} \mathrm{Pr}_{x} \mathrm{MnO}_{4}(x=0$ and 0.25$)$ samples were prepared by the so-called 'liquid-mix' or Pechini method [11]. $\mathrm{Pr}_{6} \mathrm{O}_{11}(99.9 \%$ Aldrich) was first converted into the corresponding nitrate by dissolution in diluted $\mathrm{HNO}_{3}(30 \%)$. This product was then

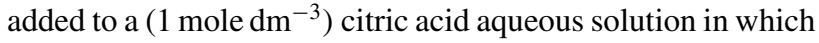
stoichiometric amounts of $\mathrm{Mn}\left(\mathrm{NO}_{3}\right)_{2} \cdot 5.3 \mathrm{H}_{2} \mathrm{O}(98 \%$, Aldrich) and $\mathrm{CaCO}_{3}$ (>99\%, Fluka) were also dissolved. After diluting the solution so obtained, we carefully added ethylene glycol in a proportion of $10 \% \mathrm{v} / \mathrm{v}$ and heated it until the formation of a brown gel that decomposed at $\sim 400^{\circ} \mathrm{C}$. The obtained ashes were given accumulative heating treatments at $600{ }^{\circ} \mathrm{C} / 24 \mathrm{~h}$ and $900^{\circ} \mathrm{C} / 48 \mathrm{~h}$, with intermediate grindings, and the pelletized samples were sintered at $1200^{\circ} \mathrm{C} / 24 \mathrm{~h}$.

X-ray powder diffraction (XRPD) patterns of all the samples were obtained with a Siemens D-5000 diffractometer and $\mathrm{Cu}(\mathrm{K} \alpha)=1.5418 \AA$ radiation. The XRPD data were analysed by the Rietveld profile analysis using the Rietica software [12]. All the samples showed a single phase with a perovskite-related $\mathrm{K}_{2} \mathrm{NiF}_{4}$ structure. These compounds display a two-dimensional structure in which perovskite blocks, that are one-octahedron thick, are separated one from 
High dielectric constant in charge-ordered $\mathrm{Ca}_{1.75} \mathrm{Pr}_{0.25} \mathrm{MnO}_{4}$

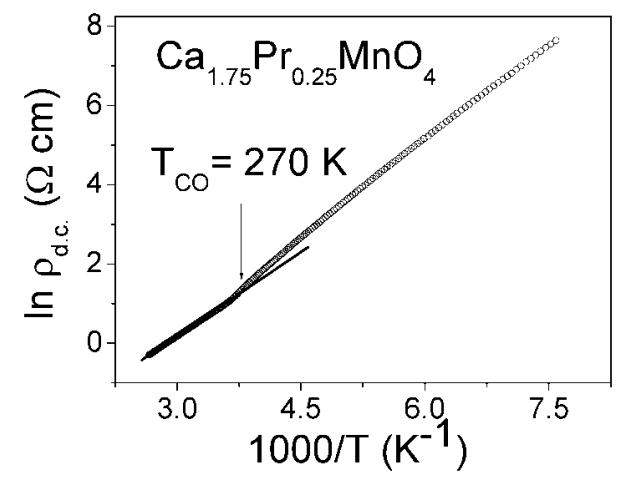

Figure 1. Plot of logarithmic dc resistivity versus inverse temperature of $\mathrm{Ca}_{1.75} \mathrm{Pr}_{0.25} \mathrm{MnO}_{4}$. The line represents the Arrhenius fit of the high temperature data and the arrow shows the $\mathrm{CO}$ temperature.

another by the presence of rock-salt-type (Pr/Ca-O) layers along the $c$-axis.

The resistivity of the samples was measured by a standard four-probe technique using a homemade device. Their complex relative dielectric permittivity $\left(\varepsilon_{\mathrm{r}}=\varepsilon_{\mathrm{r}}^{\prime}-\mathrm{i} \varepsilon_{\mathrm{r}}^{\prime \prime}\right)$ was measured with a parallel-plate capacitor coupled to a precision LCR meter (Agilent 4284 A) capable of measurements in the frequency range from $20 \mathrm{~Hz}$ to $1 \mathrm{MHz}$. The capacitor was mounted on an aluminium box refrigerated with liquid nitrogen, and incorporated a mechanism to control the temperature up to $350 \mathrm{~K}$. The samples (average surface area: $0.15-0.35 \mathrm{~cm}^{2}$ ) were prepared to fit in the capacitor, and gold was sputtered on their surfaces to ensure good electrical contact with the electrodes. In addition, to test the optimal performance of the experimental set-up, a commercial $\mathrm{SrTiO}_{3}$ sample was measured and values similar to those reported in the literature [13] were obtained. Impedance complex plane plots were analysed using the LEMV programme [14].

\section{Results and discussion}

The dc resistivity was measured in order to estimate $T_{\mathrm{CO}}$. In figure 1 we show the temperature dependence of the resistivity of $\mathrm{Ca}_{1.75} \mathrm{Pr}_{0.25} \mathrm{MnO}_{4}$, in which the arrow indicates the $\mathrm{CO}$ temperature $T_{\mathrm{CO}} \approx 270 \mathrm{~K}$, in good agreement with the value found in the literature $[10,15] . \mathrm{Ca}_{2} \mathrm{MnO}_{4}$ presents higher values, with resistivities of $9.7 \times 10^{8} \Omega \mathrm{cm}$ at $78 \mathrm{~K}$ and $1 \times 10^{4} \Omega \mathrm{cm}$ at $298 \mathrm{~K}$ [16].

Figure 2 shows the frequency dependence of the real part of the relative permittivity (dielectric constant, $\varepsilon_{\mathrm{r}}^{\prime}$ ) of $\mathrm{Ca}_{2} \mathrm{MnO}_{4}$. As it does not present a charge-order it will set the reference value. As can be seen in the curve, the dielectric permittivity decreases with frequency, whereas for a given frequency it increases with temperature. At the lowest frequency $\varepsilon_{\mathrm{r}}^{\prime}$ is strongly temperature dependent, changing from 20 at $110 \mathrm{~K}$ to $10^{4}$ at $350 \mathrm{~K}$, but this dependence reduces with frequency (it goes from 14 at $110 \mathrm{~K}$ to 70 at $350 \mathrm{~K}$ ). On the other hand, the imaginary part of the dielectric permittivity $\varepsilon_{\mathrm{r}}^{\prime \prime}$ shows rather high values, which increase almost linearly with temperature. As a result, this sample shows also loss tangents $\left(\tan \alpha=\varepsilon_{\mathrm{r}}^{\prime \prime} / \varepsilon_{\mathrm{r}}^{\prime}\right)$ that increase with temperature, with values ranging from 0.03 to 140 in the studied frequency and temperature windows.

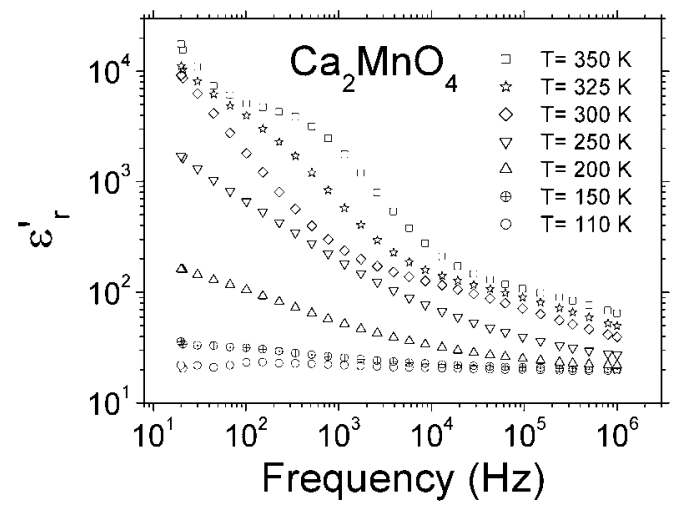

Figure 2. Frequency dependence of the $\varepsilon_{\mathrm{r}}^{\prime}$ of $\mathrm{Ca}_{2} \mathrm{MnO}_{4}$ at different temperatures.
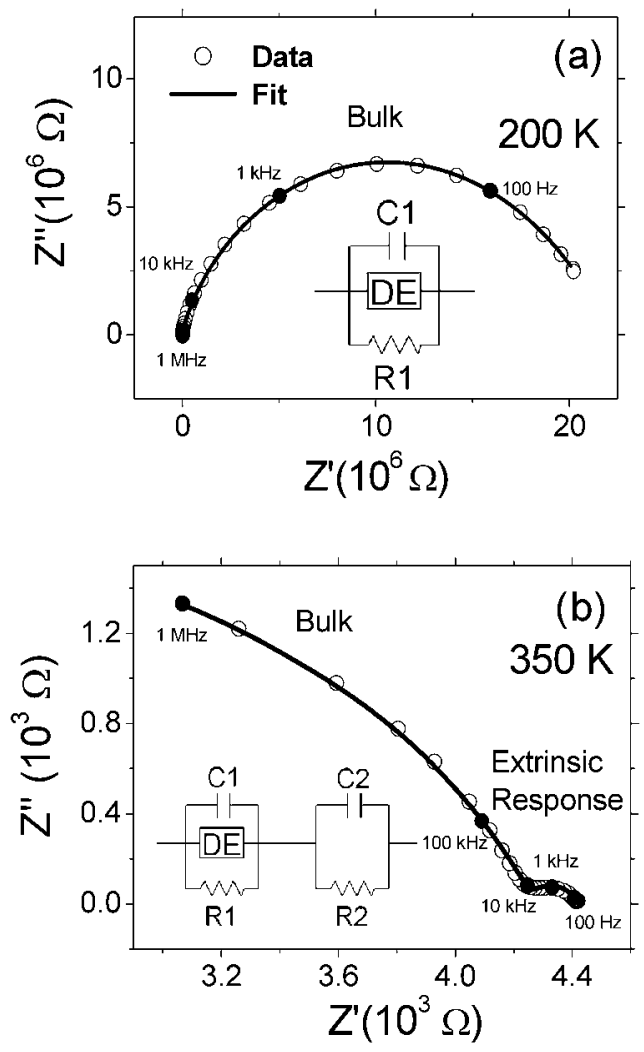

Figure 3. Examples of the two kind of $Z^{\prime \prime}$ versus $Z^{\prime}$ diagrams found in $\mathrm{Ca}_{2} \mathrm{MnO}_{4}$ : (a) lower temperature region $(110 \leqslant T(\mathrm{~K})<300)$, showing basically the arc associated to the bulk response, $(b)$ higher temperature region $(300 \leqslant T(\mathrm{~K}) \leqslant 350)$, showing two arcs attributed to the bulk and extrinsic response. The solid lines correspond to the fit of the data to the equivalent circuits shown in the figures.

The plot of the impedance complex plane diagrams of the undoped sample in the range $110-300 \mathrm{~K}$ (figure $3(a)$ ) shows a single large arc. For $T>300 \mathrm{~K}$ a second small arc appears in the low frequency range (figure $3(b)$ ). The large arc can be modelled by a circuit with three elements connected in parallel: a resistance $(R)$ and a capacitance $(C)$, that are frequency independent, and a frequency-dependent distributed element (DE). This DE is modelled as an impedance inversely proportional to a power of the frequency [17]. The 


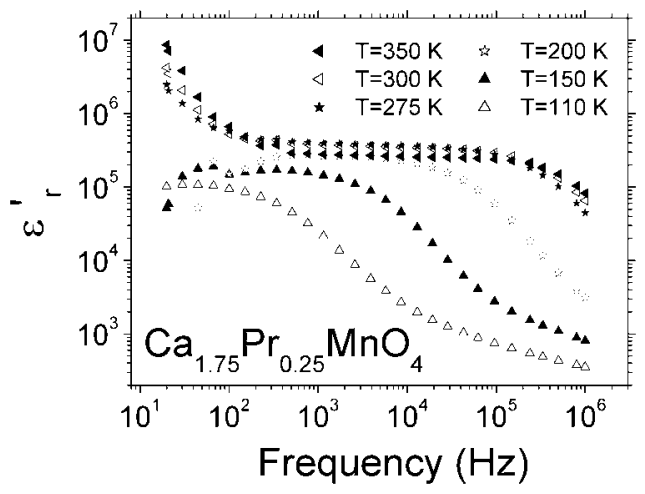

Figure 4. $\varepsilon_{\mathrm{r}}^{\prime}$ versus frequency for $\mathrm{Ca}_{1.75} \operatorname{Pr}_{0.25} \mathrm{MnO}_{4}$.

fitted frequency exponent depends slightly on temperature and for $\mathrm{Ca}_{2} \mathrm{MnO}_{4}$ is around 0.7 .

As this large arc intercepts zero (see figure 3(a)), it seems to be associated with the intrinsic (bulk) response [17]. The second arc can be modelled as a single RC connected in series with the circuit that describes the bulk arc (figure $3(b)$ ). This small arc is associated with extrinsic (barrier) contributions, like grain boundaries or electrode effects, which are easier to measure at higher temperatures, due to the decrease in resistivity.

According to these IS results, the dielectric response of $\mathrm{Ca}_{2} \mathrm{MnO}_{4}$ is mainly intrinsic in the low temperature region $T<300 \mathrm{~K}$, where $\varepsilon_{\mathrm{r}}^{\prime}$ presents lower values and it is almost frequency independent in the high frequency region. For this intrinsic dielectric constant the value calculated from the bulk capacitance is $\varepsilon_{\mathrm{r}}^{\prime}($ bulk $)=14$. Nevertheless, in the high temperature region the dielectric response is strongly affected by extrinsic factors (grain boundaries and electrode effects) due to the Maxwell-Wagner polarization.

Figure 4 shows the frequency dependence of the dielectric constant $\varepsilon_{\mathrm{r}}^{\prime}$ of $\mathrm{Ca}_{1.75} \operatorname{Pr}_{0.25} \mathrm{MnO}_{4}$. As can be observed, after an initial decrease at very low frequencies, related to diffusional processes, the high dielectric permittivity keeps a constant value for a certain frequency range, and then decreases in a step-like manner as the frequency gets higher. This behaviour is significantly different from what we find in the non-doped compound, where only a very small plateau is seen at the highest temperature in the lowest frequency region. The $\varepsilon_{\mathrm{r}}^{\prime}$ values are about two orders of magnitude higher than the values found in $\mathrm{Ca}_{2} \mathrm{MnO}_{4}$, reaching values near $10^{6}$ in the plateau. This magnification, due to extrinsic contributions, has already been observed in other compounds [18]. $\varepsilon_{\mathrm{r}}^{\prime \prime}$ shows also higher values (due to the decrease in resistivity), which increase with temperature, and it gives rise to loss tangents between 0.025 and $10^{3}$.

In order to understand the dielectric behaviour of this CO Pr-doped compound the IS analysis was also carried out. The impedance complex plane plot of $\mathrm{Ca}_{1.75} \operatorname{Pr}_{0.25} \mathrm{MnO}_{4}$ is shown in figure 5 for different temperature intervals. At low temperatures $110-200 \mathrm{~K}$, the impedance complex plane plots show two arcs. Finally, for $T>200 \mathrm{~K}$ only one arc appears and it does not intercept the origin. The number of elements in the equivalent circuit needed to simulate the impedance plane plots is different depending on the temperature. For $T<200 \mathrm{~K}$ the impedance complex plane plot can be modelled
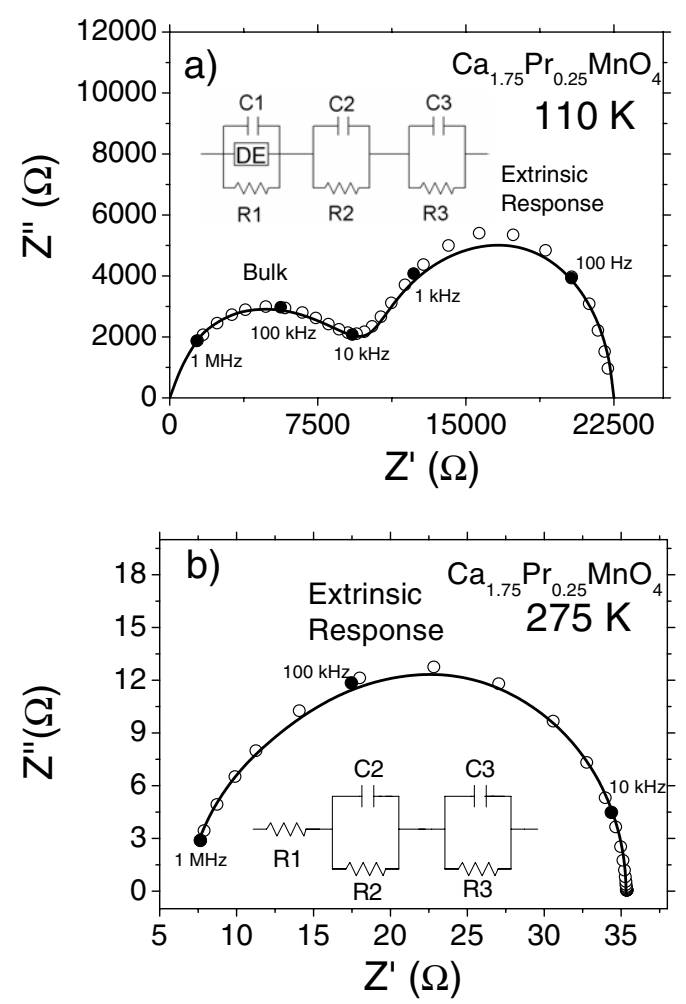

Figure 5. Examples of the two kinds of $Z^{\prime \prime}$ versus $Z^{\prime}$ diagrams found in $\mathrm{Ca}_{1.75} \mathrm{Pr}_{0.25} \mathrm{MnO}_{4}$. (a) Low temperature region $(110 \leqslant T(K) \leqslant 200)$, showing two arcs attributed to the bulk and extrinsic response, $(b)$ high temperature region $(T>200 \mathrm{~K})$, where only the arc associated to the extrinsic contributions is observed.

by a circuit with two parallel $\mathrm{RC}$ elements connected in series to a third element with $\mathrm{R}, \mathrm{C}$ and a DE connected in parallel, with a frequency exponent 0.6. In figure $5(a)$ we show the example of the fit at $110 \mathrm{~K}$. The order of magnitude of the capacitance associated with the high frequency arc is of picofarads per centimetre, typical of bulk response [17], whereas the capacitance of the low frequency arc is of the order of magnitude of nanofarads per centimetre, typical of extrinsic effects [17].

In the high temperature range, the impedance complex plane plots show an arc which does not intercept the coordinates origin (figure $5(b)$ ), which means that at these temperatures and in the studied frequency range only the extrinsic contribution is observed. This plot can be modelled with two RC elements connected in series and one R, in order to consider the resistance of the non-observed bulk. The two parallel RC elements simulate the extrinsic contributions (grain and electrodes boundaries) whereas the third element simulates the bulk contribution. This circuit is similar to the one used in the high temperature region of $\mathrm{Ca}_{2} \mathrm{MnO}_{4}$, but in the case of Pr-doped samples we have to add a new RC to fit the data attributed to extrinsic factors. In fact, if we plot the measured $\varepsilon_{\mathrm{r}}^{\prime}$ versus $T$ (figure 6), the $\varepsilon_{\mathrm{r}}^{\prime}$ value is mainly intrinsic at the lowest temperatures. But at higher temperatures $T>150 \mathrm{~K}$, there is a notable rise in the permittivity probably due to the influence of extrinsic factors, which does not allow us to obtain clear information about the behaviour of the intrinsic dielectric response at the charge ordering temperature. In this region, we do not have data to estimate the bulk capacitance, and we 


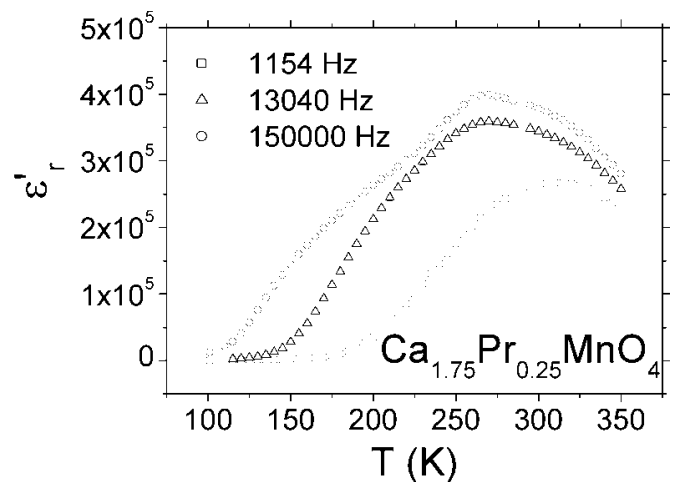

Figure 6. Temperature dependence of the dielectric permittivity of $\mathrm{Ca}_{1.75} \operatorname{Pr}_{0.25} \mathrm{MnO}_{4}$, showing the dramatic increase in $\varepsilon_{\mathrm{r}}^{\prime}$ with increasing temperature, measured at different frequencies.

cannot obtain information about the intrinsic $\varepsilon_{\mathrm{r}}^{\prime}$ (bulk) value at the charge ordering temperature. The shape of the curves in figure 6 , with maxima that shift to higher temperatures with increasing frequency, indicates dielectric relaxation.

The intrinsic dielectric constant calculated from the values of the obtained bulk capacitances is 217 . Taking into account that in these type of oxides $\varepsilon_{\mathrm{r}}^{\prime}$ is of the order of 10 [19] the value obtained here is remarkably high, even more if we take into account that it is not a ferroelectric material.

Moreover, if we compare it with the $\varepsilon_{\mathrm{r}}^{\prime}$ of the non$\mathrm{CO}$ compound $\mathrm{Ca}_{2} \mathrm{MnO}_{4}\left(\varepsilon_{\mathrm{r}}^{\prime}=14\right)$, these results suggest that the $\mathrm{CO}$ state may play an important role in this increase in the dielectric constants. The value obtained in the non-CO compound $\mathrm{Ca}_{2} \mathrm{MnO}_{4}$ is expected taking into account the electronic polarizability of its cations [19], but the $\mathrm{CO} \mathrm{Ca}_{1.75} \operatorname{Pr}_{0.25} \mathrm{MnO}_{4}$ seems to present a extra ionic polarizability which increases the value of the dielectric constant. The explanation to this unexpected increase in the dielectric constant could be in the recent idea of Efremov, van den Brink and Khomskii [20], who have recently reported that in $\mathrm{CO}$ and orbitally-ordered perovskites there is a stable range of coexistence of two types of chargeordering: site-centred and bond-centred. The combination of both yields an intermediate phase which lacks inversion symmetry and would be ferroelectric. The first experimental observation of this bond-centred charge-ordering has been given by Daoud-Aladine et al [21] in $\operatorname{Pr}_{0.60} \mathrm{Ca}_{0.40} \mathrm{MnO}_{3}$ (very similar to the prototypical $\operatorname{Pr}_{2 / 3} \mathrm{Ca}_{1 / 3} \mathrm{MnO}_{3}$ ). Maybe further work completing that of Ibarra et al [10] and Autret et al [15] (which interpret the incommensurate chargeorder pattern of $\mathrm{Ca}_{2-x} \mathrm{Pr}_{x} \mathrm{MnO}_{4}$ as partial delocalization, involving $\mathrm{Mn}^{(3+\delta)+}$ and $\mathrm{Mn}^{(4-\delta)+}$ stripes) could yield a similar result.

In order to confirm these results, the dielectric constant of $\mathrm{Ca}_{1.75} \operatorname{Pr}_{0.25} \mathrm{MnO}_{4}$ was also obtained graphically (figure 7), by using the expression $\varepsilon_{\mathrm{r}}^{\prime}=\varepsilon_{\mathrm{r}}^{\prime}\left(\right.$ bulk) $+\mathrm{A} \omega^{s-1}$ (where $A=$ $\left.\left(\sigma_{0} / \varepsilon_{0}\right) \tan (s \pi / 2)\right)[22] ; \sigma_{0}$ is generally weakly temperature dependent, $s \leqslant 1$ is the exponent of the power law and $\omega=2 \pi f$ is the angular frequency). These plots of $\varepsilon_{\mathrm{r}}^{\prime}$ versus $\omega^{s-1}$ at fixed temperatures yield $\varepsilon_{\mathrm{r}}^{\prime}$ (intrinsic) as the common intercept (i.e. in the limit $\omega \rightarrow \infty$ ). At each temperature, $s$ is determined from the frequency dependence of the conductivity $\sigma_{\mathrm{d}}=\sigma_{\mathrm{dc}}+\sigma_{\mathrm{o}} \omega^{s}$ (universal dielectric response) where, $\sigma_{\mathrm{dc}}$ is

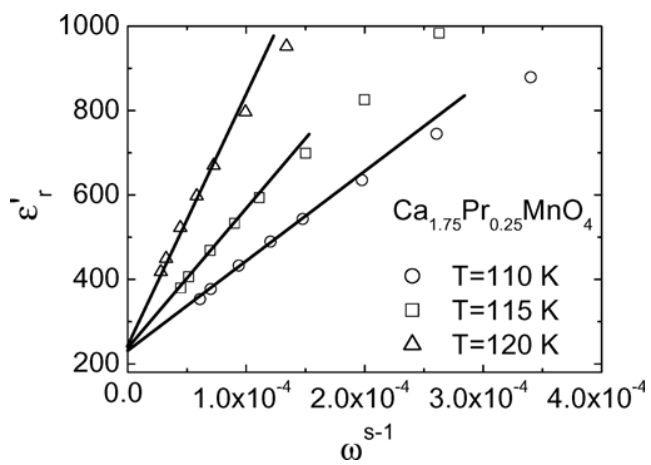

Figure 7. $\varepsilon_{\mathrm{r}}^{\prime}$ versus $\omega^{s-1}$ curves for $\mathrm{Ca}_{1.75} \operatorname{Pr}_{0.25} \mathrm{MnO}_{4}$ obtained at low temperatures.

the dc conductivity. By this method, we obtain a value of $\varepsilon_{\mathrm{r}}^{\prime}=221$, which confirms quite well the value calculated by the previous method.

\section{Conclusions}

We have studied the dielectric properties of $\mathrm{Ca}_{2} \mathrm{MnO}_{4}$ and $\mathrm{Ca}_{1.75} \mathrm{Pr}_{0.25} \mathrm{MnO}_{4}$, in the search for differences depending on the presence or absence of charge-order. Namely, for the non-CO compound $\mathrm{Ca}_{2} \mathrm{MnO}_{4}$ the intrinsic dielectric constant is 14 , whereas for the $\mathrm{CO}$ compound $\mathrm{Ca}_{1.75} \operatorname{Pr}_{0.25} \mathrm{MnO}_{4}$ it is $\sim 220$, much higher. This suggests a direction for finding materials with strong dielectric behaviour, considering those with condensation of electronic charge.

\section{Acknowledgments}

The authors are grateful for financial support given by DGICYT, Ministerio de Educación y Ciencia of Spain and UE (FEDER), under project MAT2004-05130.

\section{References}

[1] Salamon M B and Jaime M 2001 Rev. Mod. Phys. 73583

[2] Jardón C, Rivadulla F, Hueso L E, Fondado A, Rivas J, López Quintela M A, Zysler R, Causa M T and Sande P 1999 J. Magn. Magn. Mater. 196-197 475

[3] Rivadulla F, López Quintela M A, Hueso L E, Jardón C, Fondado A, Rivas J, Causa M T and Sánchez R D 1999 Solid State Commun. 110179

[4] Saraf L V, Ogale S B, Chen Z, Godfrey R P, Venkatesan T and Ramesh R 2000 Phys. Rev. B 62 R11961

[5] Mercone S, Wahl A, Pautrat A, Pollet M and Simon C 2004 Phys. Rev. B 69174433

[6] Subramanian M A, Li D, Duan N, Reisner B A and Sheight A W 2000 J. Solid State Chem. 151323

[7] Sinclair D C, Adams T B, Morrison F D and West A R 2002 Appl. Phys. Lett. 802153

[8] Lunkenheimer P, Bobnar V, Pronin A V, Ritus A I, Volkov A A and Loidl A 2002 Phys. Rev. B 6652105

[9] Lunkenheimer P, Fichtl R, Ebbinghaus S G and Loidl A 2004 Phys. Rev. B 70172102

[10] Ibarra M, Retoux R, Hervieu M, Autret C, Maignan A, Martin C and Raveau B 2003 J. Solid State Chem. 170 361

[11] Licci F, Besagni T and Rinaldi L 1985 European Patent Appl. No. 85860253-2 
[12] Howard J, Hunter H and Rietica B A 1997 A Computer Program for Rietveld Analysis of X-ray and Neutron Powder Diffraction Patterns, Australian Nuclear Science and Technology Organization, Lucas Heights Research Laboratories

[13] Takashima H, Wang R, Kasai N and Shoji A 2003 Appl. Phys. Lett. 832883

[14] Ross Macdonald J 2003 LEVM version 8.0 Complex Nonlinear Squares Fitting Program

[15] Autret C, Retoux R, Hervieu M and Raveau B 2001 Chem. Mater. 134745

[16] MacChesney J B, Williams H J, Potter J F and Sherwood R C 1967 Phys. Rev. B 164779
[17] Ross Macdonald J 1987 Impedance Spectroscopy Emphasizing Solid Materials and Systems (New York: Wiley)

[18] Rivas J, Rivas-Murias B, Fondado A, Mira J and Señarís Rodríguez M A 2004 Appl. Phys. Lett. 85 6224

[19] Shannon R D 1993 J. Appl. Phys. 73348

[20] Efremov D V, van den Brink J and Khomskii D I 2004 Nature Mater. 3853

[21] Daoud-Aladine A, Rodríguez-Carvajal J, Pinsard-Gaudard L, Fernández-Díaz M T and Revcolevschi A 2002 Phys. Rev. Lett. 89097205

[22] Jonscher A K 1983 Dielectric Relaxation in Solids (London: Chelsea Dielectric Press) 